1988-01-01

\title{
Quasilinear evolution equations in nonclassical diffusion
}

Kenneth Kuttler

Elias Aifantis

Follow this and additional works at: https://scholarsarchive.byu.edu/facpub

Part of the Mathematics Commons

Original Publication Citation

SIAM Journal on Mathematical Analysis, 19.1 (1988), 11-12.

\section{BYU ScholarsArchive Citation}

Kuttler, Kenneth and Aifantis, Elias, "Quasilinear evolution equations in nonclassical diffusion" (1988). Faculty Publications. 740.

https://scholarsarchive.byu.edu/facpub/740 


\title{
QUASILINEAR EVOLUTION EQUATIONS IN NONCLASSICAL DIFFUSION*
}

\author{
KENNETH KUTTLER $†$ AND ELIAS AIFANTIS $\ddagger$
}

\begin{abstract}
After describing the motivation leading to some nonclassical diffusion equations, we formulate a general abstract nonlinear evolution equation and establish existence of solutions. Then we return to the original equation and discuss particular initial-boundary value problems.
\end{abstract}

Key words. existence, modeling

AMS(MOS) subject classifications. 35A05, 35G30, 35K60, 35K70

Introduction. A general framework based on the approach of continuum mechanics has been proposed recently by Aifantis [1] for a systematic development of diffusion models. In this method, the diffusing substance is viewed as a continuum subject to two kinds of forces: an internal body force vector arising from its interaction with the matrix and a stress tensor that the diffusing substance exerts on itself.

By introducing constitutive equations for the stress tensor and the internal force vector, we can obtain classes of diffusion behavior which take into account viscosity and higher-order gradient effects. Various diffusion models are thus generated within a unified mathematical framework.

For example, if the stress tensor is assumed to depend on the concentration and the gradient of the flux, while the internal body force is viewed as a drag proportional to the flux, a pseudoparabolic partial differential equation of the type studied by Ting [2] is obtained. This yields a physically realistic model of diffusion for situations where the effects of viscosity cannot be ignored. Similarly, the equation of spinodal decomposition of Cahn [3] can be obtained within this general formalism by including second gradients of the solute concentration in the constitutive equation for the stress tensor to allow for long-range effects. For a further discussion of the method and the development of many other examples, we refer to [1].

A central problem in the development of these new models is to determine which of the resulting partial differential equations are well posed. This is not always obvious, especially if nonlinear or time dependent equations are being considered. In a preceding paper [4], the questions of existence and uniqueness were resolved for a class of linear partial differential equations resulting when the stress $\underset{\sim}{T}$ is a linear function of the concentration, its gradients up to second order, and the gradient of the flux, while the internal body force $f$ is a linear function of the flux. The corresponding constitutive equations are thus

$$
\begin{aligned}
& \underset{\sim}{T}=c_{1} \rho \underset{\sim}{1}+c_{2} \operatorname{tr}(\nabla \underset{\sim}{j}) \underset{\sim}{1}+c_{3} \operatorname{tr}\left(\nabla^{2} \rho\right) \underset{\sim}{1}, \\
& \underset{\sim}{f}=-\underset{\sim}{\alpha}(\underset{\sim}{x}, t) \underset{\sim}{j},
\end{aligned}
$$

where $j$ is the flux, $\rho$ is the concentration, $\nabla j$ denotes the first gradient of $j\left(\nabla j=j_{i, j}\right)$, $\nabla^{2} \rho$ the second gradient of $\rho\left(\nabla^{2} \rho=\rho_{, i j}\right)$, tr is the trace and $\underset{\alpha}{\alpha}(\underset{x}{,}, t)$ is a nonsingular

* Received by the editors February 4, 1985; accepted for publication December 1, 1986. This work was supported by the Michigan Technological University creativity grants program, the SM program of the National Science Foundation and the MM program of Michigan Technological University.

$\dagger$ Department of Mathematical and Computer Sciences, Michigan Technological University, Houghton, Michigan 49931.

¥Department of Mechanical Engineering and Engineering Mechanics, Michigan Technological University, Houghton, Michigan 49931. 
symmetric matrix. The spatial and temporal dependence of $\alpha$ models the inhomogeneity of the interaction between the solid and the diffusing substance. The reason for neglecting nonhydrostatic components in the expression for the stress tensor in $(0,1)_{1}$ is also discussed in [4].

Next we introduce $(0.1)$ into the balance equations of mass and momentum which, on neglecting inertia forces, take the form

$$
\begin{aligned}
& \rho_{t}+\operatorname{div} \underset{\sim}{j}=0 \\
& \operatorname{div} \underset{\sim}{f}+\underset{\sim}{f}=0
\end{aligned}
$$

This operation yields the following linear evolution equation whose existence and uniqueness have been studied earlier [4].

$$
\frac{\partial}{\partial t}\left(\rho-c_{2} \operatorname{div}\left(\underline{\alpha}^{-1} \nabla \rho\right)\right)=-c_{1} \operatorname{div}\left({\underset{\alpha}{\alpha}}^{-1} \nabla \rho\right)-c_{3} \operatorname{div}\left({\underset{\alpha}{\alpha}}^{-1} \nabla(\Delta \rho)\right)
$$

In the present paper we consider a more general physical situation by allowing the constants $c_{1}$ and $c_{3}$ in $(0.1)_{1}$ to be functions of $\rho$. Roughly speaking, this means physically that we consider situations where the diffusion coefficient is concentration dependent. In this connection, our results are most suitable for problems in the nonlinear theory of spinodal decomposition, where $c_{2}$ vanishes identically. Thus our present expression for the stress $\underset{\sim}{T}$ takes the form

$$
T=c_{1}(\rho) \underset{\sim}{1}+c_{2} \operatorname{tr}(\nabla \underset{\sim}{j}) \underset{\sim}{1}+c_{3}(\rho) \operatorname{tr}\left(\nabla^{2} \rho\right) \underset{\sim}{1} .
$$

On substituting $(0.4)$ in the balance laws $(0.2)$, we find that $\rho$ satisfies a partial differential equation of the form

$$
\rho_{t}-c_{2} \operatorname{div}\left(\underset{\sim}{\beta}(x, t) \nabla \rho_{t}\right)+\operatorname{div}\left(\underset{\sim}{\beta} \nabla\left(c_{3}(\rho) \Delta \rho\right)\right)+\operatorname{div}\left(\underset{\sim}{\beta} \nabla c_{1}(\rho)\right)=0
$$

where $\beta(\underset{\sim}{x}, t)=\alpha^{-1}(x, t)$. As explained in [4], we could also have allowed $c_{1}, c_{2}$ and $c_{3}$ to depend on $x$ and $t$, but in any case, we would have arrived at an equation of the following general form:

$$
\begin{aligned}
\frac{\partial}{\partial t}\left(\rho-\sum_{i j} \partial_{i}\left(\tilde{D}_{i j}(x, t) \partial_{j} \rho\right)\right)-\sum_{i j} \partial_{i}\left(D_{i j}(x, t ; \rho) \partial_{j} \rho\right) \\
+\sum_{|\alpha|,|\beta| \leqq 2}(-1)^{|\beta|} D^{\beta}\left(E_{\alpha \beta}(x, t ; \rho) D^{\alpha} \rho\right)=h(x, t),
\end{aligned}
$$

where $\alpha, \beta$ are multi-indices [5], $h$ is a source function and the rest of the coefficient functions are to be specified later. Since this extra generality does not create essential difficulties in the mathematical treatment, we will consider this last equation. We show that weak solutions to (0.6) exist by formulating a corresponding abstract problem and obtaining estimates that allow the use of a fixed point theorem.

The plan of the paper is as follows: Section 1 is a review of the linear version of these equations. Section 2 contains an abstract result, motivated by (0.6), which might be useful in other problems in the theory of nonlinear evolution equations. This result is used in $\S 3$ to reduce the question of existence of weak solutions for $(0.6)$ to the verification of a coercivity inequality. In $\S 4$ we actually prove that this inequality holds. This way, we obtain the existence of solutions for (0.6) to some specific initial-boundary value problems. We use the standard notation for Banach spaces: If $V$ is a Banach space, $V^{\prime}$ denotes its dual, $\rightarrow$ denotes strong convergence, and $\rightarrow$ denotes weak convergence. If $i: V \rightarrow W$ is an injection map, $i^{*}$ denotes the dual map defined by

$$
\left\langle i^{*} f, u\right\rangle=\langle f, i u\rangle=\langle f, u\rangle \text {. }
$$


1. The linear equations. Before dealing with $(0.6)$, we discuss briefly the main results of the earlier paper [4] in which $D_{i j}$ and $E_{\alpha \beta}$ do not depend on $\rho$. In this earlier paper, the assumptions were

$$
\begin{aligned}
& \tilde{D}_{i j}=\tilde{D}_{j i}, \\
& \tilde{D}_{i j} \quad \text { is bounded, measurable and } C^{1} \text { in } t, \\
& \sum_{i j} \tilde{D}_{i j} \xi_{i} \xi_{j} \geqq 0 \text { for } \xi=\left(\xi_{1}, \xi_{2}, \xi_{3}\right) \in R^{3}, \\
& D_{i j} \in L^{\infty}(\Omega \times[0, T]), \\
& E_{\alpha \beta} \in L^{\infty}(\Omega \times[0, T]),
\end{aligned}
$$

along with a coercivity inequality similar to (2.5) of the present paper.

Under these assumptions, existence, uniqueness and continuous dependence results were obtained for a large class of initial-boundary value problems associated with the linear version of (0.6). The existence part was based on the verification of this coercivity inequality which allowed the use of the main existence theorem of [6] or [11]. The uniqueness may be obtained as a special case of the uniqueness theorem of $[6]$.

To be more specific, sufficient conditions were given for well-posedness of weak solutions of the following initial boundary value problems:

$$
\begin{aligned}
& \frac{\partial}{\partial t}\left(\rho-\sum_{i j} \partial_{i}\left(\tilde{D}_{i j}(x, t) \partial_{j} \rho\right)\right)-\sum_{i j} \partial_{i}\left(D_{i j}(x, t) \partial_{j} \rho\right)+\Delta^{2} \rho=g(x, t), \\
& \rho(x, 0)=\rho_{0}(x),
\end{aligned}
$$

along with either the boundary conditions

$$
\begin{aligned}
& \frac{\partial \rho}{\partial n}(x, t)=\frac{\partial w}{\partial n}(x, t), \quad x \in \partial \Omega, \\
& \frac{\partial}{\partial t}\left(\sum_{i j} \tilde{D}_{i j} \partial_{j} \rho n_{i}\right)+\sum_{i j}\left(D_{i j} \partial_{i} \rho\right) n_{i}-\frac{\partial(\Delta \rho)}{\partial n}=l, \quad x \in \partial \Omega,
\end{aligned}
$$

or the boundary conditions

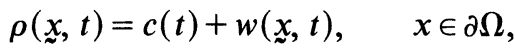

$$
\begin{aligned}
& \int_{\partial \Omega} \frac{\partial \rho}{\partial t}(x, t) d s=\int_{\partial \Omega} \frac{\partial w}{\partial n}(x, t) d s, \\
& \int_{\partial \Omega}\left[\frac{\partial}{\partial t}\left(\sum_{i j} \tilde{D}_{i j} \partial_{j} \rho\right) n_{i}+\sum_{i j} D_{i j} \partial_{j} \rho n_{i}-\frac{\partial(\Delta \rho)}{\partial n}\right] d s=\int_{\partial \Omega} l(x, t) d s, \\
& \Delta \rho(x, t)-k(x, t)=r(t)
\end{aligned}
$$

where in (1.2.3)-(1.2.8), $r(t), c(t)$ are unknown functions and $w$ and $l$ are given functions.

In the present paper, we shall use the existence and uniqueness of solutions to an appropriate abstract version of the linear problem, along with a well-known generalization of the Brouer fixed point theorem, to establish the existence of solutions to initial-boundary value problems corresponding to (0.6). We shall show that, just as in the linear case, the verification of an appropriate inequality is sufficient. 
2. The abstract equation. For the sake of both generality and simplicity in the presentation, we obtain existence of solutions to (0.6) as a special case of an abstract result. We introduce the following hypotheses and conventions:

$$
\begin{aligned}
& V, W \text { are reflexive Banach spaces } V \subseteq W,\|v\|_{V} \geqq\|v\|_{W} \text {, so that } \\
& V \subseteq W \subseteq i^{*} W^{\prime} \subseteq V^{\prime} .
\end{aligned}
$$

On defining $B(t)$ as a continuous linear map from $W$ to $W^{\prime}$, we will assume

$$
\begin{aligned}
& \langle B(t) u, u\rangle \geqq 0, \\
& \langle B(t) u, v\rangle=\langle B(t) v, u\rangle, \\
& t \rightarrow B(t) u \quad \text { is in } C^{1}\left(0, T ; W^{\prime}\right) .
\end{aligned}
$$

We will also make use of the space

$$
\begin{aligned}
& X=\left\{u \in L^{2}(0, T ; V)\right. \text { such that } \\
& \left.\qquad(B u)^{\prime} \in L^{2}\left(0, T ; V^{\prime}\right)\right\}\|u\|_{X}=\|u\|_{L^{2}(0, T ; V)}+\left\|(B u)^{\prime}\right\|_{L^{2}\left(0, T ; V^{\prime}\right)}
\end{aligned}
$$

where by $(B u)^{\prime}$ we mean a unique function in $L^{2}\left(0, T ; V^{\prime}\right)$, such that

$$
\int_{0}^{T}(B u)^{\prime}(t) \phi(t) d t=-\int_{0}^{T} i^{*} B(t) u(t) \phi^{\prime}(t) d t \text { for all } \phi \in C_{0}^{\infty}(0, T) .
$$

It follows that $X$ is a reflexive Banach space.

For each $w \in L^{2}(0, T ; V)$, let $A(w)$ be a continuous linear map from $L^{2}(0, T ; V)$ to $L^{2}\left(0, T ; V^{\prime}\right)$ satisfying the following property:

(i) $\sup \left\{\|A(w)\|, w \in L^{2}(0, T ; V)\right\}=Q<\infty$,

(ii) If $u_{n} \rightarrow u$ in $X$ and $v_{n} \rightarrow v$ in $L^{2}(0, T ; V)$ then for some subsequence

$$
u_{n^{\prime}}, v_{n^{\prime}}, A\left(u_{n^{\prime}}\right) v_{n^{\prime}} \rightarrow A(u) v \text { in } L^{2}\left(0, T ; V^{\prime}\right) \text {. }
$$

Moreover, by introducing the definitions

(i) $B: L^{2}(0, T ; W) \rightarrow L^{2}\left(0, T ; W^{\prime}\right)$ is given by $B(t) u(t)=B u(t)$,

(ii) $B^{\prime}: L^{2}(0, T ; W) \rightarrow L^{2}\left(0, T ; W^{\prime}\right)$ is given by $B^{\prime}(t) u(t)=B^{\prime} u(t)$, we can postulate the following coercivity inequality:

$$
2\langle A(w) u, u\rangle+\lambda\langle B u, u\rangle+\left\langle B^{\prime} u, u\right\rangle \geqq C_{1}\|u\|_{L^{2}(0, T ; V)}^{2},
$$

for some $\lambda \in \mathbb{R}$ independent of $w$ and $C_{1}>0$.

Finally, for each $u \in L^{2}(0, T ; V)$, let $f(u) \in L^{2}\left(0, T ; V^{\prime}\right)$ satisfy the following properties:

(i) $\sup \left\{\|f(u)\|_{L^{2}\left(0, T ; V^{\prime}\right)}, u \in X\right\}=P<\infty$,

(ii) If $u_{n} \rightarrow u$ in $X$, then $f\left(u_{n^{\prime}}\right) \rightarrow f(u)$ in $L^{2}\left(0, T ; V^{\prime}\right)$, for some subsequence $u_{n^{\prime}}$.

With these assumptions, we can state the main existence theorem.

Theorem 1. With (2.1)-(2.6) valid and $u_{0} \in V$, there exists $u \in X$ such that

$$
\begin{aligned}
& (B u)^{\prime}+A(u) u=f(u), \\
& i^{*} B u(0)=i^{*} B(0) u_{0} .
\end{aligned}
$$


Proof. It follows from (2.5) and [6] that for each $w \in X$ there exists a unique solution $u \in X$ to the problem

$$
\begin{aligned}
& (B u)^{\prime}+A(w)(u)=f(w), \\
& i^{*} B u(0)=i^{*} B(0) u_{0} .
\end{aligned}
$$

On denoting this solution by $\psi(w)$ we have $\psi: X \rightarrow X$.

Next we make use of three lemmas whose proof may be found in [6].

LEMMA 1. For each $u \in X$,

$$
\left\langle(B u)^{\prime}(t), u(t)\right\rangle=\frac{1}{2}\left[\frac{d}{d t}\langle B u(t), u(t)\rangle+\left\langle B^{\prime}(t) u(t), u(t)\right\rangle\right] \text { a.e. }
$$

Moreover $\langle B u(t), u(t)\rangle$ is equal to an absolutely continuous function a.e. and point evaluation of $i^{*} \mathrm{Bu}(\cdot)$ is a continuous map from $X$ to $V^{\prime}$.

LEMMA 2. For $u, v \in X,\langle B u(t), v(t)\rangle$ equals an absolutely continuous function a.e. denoted by $\langle B u, v\rangle(\cdot)$. Moreover, there exists a constant $M$ such that

$$
|\langle B u, v\rangle(t)| \leqq M\|u\|_{X}\|v\|_{X} \quad \text { for all } t \in[0, T] .
$$

LeMma 3. If $i^{*} B v(0)=0$ for $v \in X$, then there exists a sequence $\left\{v_{n}\right\} \subseteq X$ such that $\left\|v-v_{n}\right\|_{X} \rightarrow 0$ and $v_{n}(t)=0$ in some neighborhood of 0 .

As a consequence of Lemmas 1-3 we can establish the following results:

(i) $\langle B u, u\rangle(t)-\langle B u, u\rangle(0)+\int_{0}^{t}\left\langle B^{\prime}(s) u(s), u(s)\right\rangle d s$

$$
+2 \int_{0}^{t}\langle A(w) u(s), u(s)\rangle d s=2 \int_{0}^{t}\langle f(w)(s), u(s)\rangle d s,
$$

$$
\text { (ii) }\langle B u, u\rangle(0)=\left\langle B(0) u_{0}, u_{0}\right\rangle
$$

Relation (2.11) is obtained by multiplying (2.8) $)_{1}$ by $u$, using Lemma 1 and integrating the result from 0 to $t$. Relation (2.12) is derived by first using Lemma 3 to obtain a sequence $\left\{u_{n}\right\} \subseteq X$ with $u_{n}(t)=u_{0}$ near 0 and converging to $u$ in $X$, and then using Lemma 2 together with the inequality

$$
\begin{aligned}
\mid\langle B u, u\rangle(0)-\left\langle B(0) u_{0}, u_{0}\right\rangle & =\left|\langle B u, u\rangle(0)-\left\langle B u_{n}, u_{n}\right\rangle(0)\right| \\
& \leqq\left|\left\langle B\left(u_{n}-u\right), u_{n}\right\rangle(0)\right|+\left|\left\langle B u, u_{n}-u\right\rangle(0)\right| \\
& \leqq M\left(\|u\|_{X}+\left\|u_{n}\right\|_{X}\right)\left\|u_{n}-u\right\|_{X} .
\end{aligned}
$$

With (2.11) and (2.12) valid and the use of (2.5) and (2.6), we can establish the following main inequality:

$$
\begin{aligned}
& \langle B u, u\rangle(t)+C_{1}\|u\|_{L^{2}(0, t ; V)}^{2} \\
& \quad \leqq \lambda \int_{0}^{t}\langle B u(s), u(s)\rangle d s+2 P\|u\|_{L^{2}(0, t ; V)}+\left\langle B(0) u_{0}, u_{0}\right\rangle
\end{aligned}
$$

On subtracting $C_{1}\|u\|_{L^{2}(0, t ; V)}^{2}$ from both sides, we first note that

$$
2 P\|u\|_{L^{2}(0, t ; V)}-C_{1}\|u\|_{L^{2}(0, t ; V)}^{2} \leqq \frac{P^{2}}{C_{1}} .
$$

It then follows that

$$
\langle B u, u\rangle(t) \leqq\left(\left\langle B(0) u_{0}, u_{0}\right\rangle+\frac{P^{2}}{C_{1}}\right) e^{\lambda t}
$$


by an application of Gronwall's inequality. Having thus established that $\langle B u, u\rangle(t)$ is bounded uniformly for $t \in[0, T]$ independently of $w,(2.14)$ implies that $\|\psi w\|_{L^{2}(0, T ; V)}$ is bounded independently of $w$. It now follows from $(2.8)_{1},(2.4)$ and (2.6) that $\|\psi w\|_{X}$ is bounded independently of $w$. Moreover, if $N \geqq \sup \left\{\|\psi w\|_{X}, w \in X\right\}$ and $S=\{w \in X$ such that $\left.\|w\|_{X} \leqq N\right\}$, it follows that $\psi: X \rightarrow S$.

As a final step in the proof of the theorem we establish the following.

LeMmA 4. $\psi: X \rightarrow X$ is weakly continuous.

Proof. Let $u_{n} \rightarrow u$ in $X$. If $\psi u_{n}$ fails to converge weakly to $\psi u$, then by selecting a subsequence also denoted by $u_{n}$, we may assume $u_{n} \rightarrow u$ in $X$ and $\psi u_{n} \rightarrow z \neq \psi u$ in $X$. By utilizing the definition of $\psi$

$$
\begin{aligned}
& \left(B\left(\psi u_{n}\right)\right)^{\prime}+A\left(u_{n}\right) \psi u_{n}=f\left(u_{n}\right), \\
& i^{*} B\left(\psi u_{n}\right)(0)=i^{*} B(0) u_{0},
\end{aligned}
$$

and properties (2.4) and (2.6) together with Lemma 1, we obtain

$$
\begin{aligned}
& (B z)^{\prime}+A(u) z=f(u), \\
& i^{*} B z(0)=i^{*} B(0) u_{0} .
\end{aligned}
$$

Obviously, (2.18) contradicts the assumption that $z \neq \psi u$; therefore $\psi$ is weakly continuous and Lemma 4 is established.

Thus, the proof of Theorem 1 is now completed by invoking Tykhanov's fixed point theorem [7] which asserts that $\psi$ has a fixed point in $S$.

3. The nonlinear partial differential equation. Here we apply the abstract result of $\S 2$ to the question of existence of solutions for initial-boundary value problems associated with the generalized diffusion equation (0.6). We will assume the following general properties for the relevant coefficients:

(i) $\tilde{D}_{i j}=\tilde{D}_{j i}$,

(ii) $\tilde{D}_{i j}$ is bounded, measurable, and $C^{1}$ in $t$,

$$
\begin{aligned}
& \text { (iii) } \sum_{i j} \tilde{D}_{i j} \xi_{i} \xi_{j} \geqq 0 \quad \text { for } \xi=\left(\xi_{1}, \xi_{2}, \xi_{3}\right) \in \mathbb{R}^{3}, \\
& \text { (iv) } \sup \left\{\sum_{i j}\left|D_{i j}(t, x ; r)\right|+\sum_{|\alpha|,|\beta| \leqq 2}\left|E_{\alpha \beta}(x, t ; r)\right|,(x, t, r) \in \bar{\Omega} \times[0, T] \times \mathbb{R}\right\}<\infty,
\end{aligned}
$$

(v) $\quad r \rightarrow D_{i j}(x, t ; r)$ and $r \rightarrow E_{\alpha \beta}(x, t ; r)$ are continuous and real valued

where $\Omega$ is a bounded open set in $\mathbb{R}^{3}$.

With these, and in order to cast (0.6) in the abstract form of (2.7), we let $V$ be a closed subspace of $H^{2}(\Omega), W=H^{1}(\Omega), H=L^{2}(\Omega)$, and for $y \in L^{2}\left(0, T ; H^{2}(\Omega)\right)$ we let $\tilde{A}(y)$ be a continuous linear map from $L^{2}(0, T ; V)$ to $L^{2}\left(0, T ; V^{\prime}\right)$ defined by

$$
\begin{aligned}
\langle\tilde{A}(y) u, v\rangle=\int_{0}^{T} \int_{\Omega} D_{i j}(x, t ; y(t)(x)) \partial_{i} u(t)(x) \partial_{j} v(t)(x) d x d t \\
\quad+\int_{0}^{T} \int_{\Omega} E_{\alpha \beta}(x, t ; y(t)(x)) D^{\alpha} u(t)(x) D^{\beta} v(t)(x) d x d t,
\end{aligned}
$$

and $B(t) ; W \rightarrow W^{\prime}$ defined by

$$
\langle B(t) u, v\rangle=\int_{\Omega} u(x) v(x)+\tilde{D}_{i j}(x, t) \partial_{i} u(x) \partial_{j} v(x) d x,
$$


where summation over repeated indices is assumed. With $B(t)$ given by (3.3), it is clear that (2.2) holds.

Having already specified definitions and hypotheses (2.1)-(2.3), we proceed by considering properties (2.4)-(2.6). Of these properties, (2.4) and (2.6) are verified in this section while the coercivity inequality $(2.5)$ is examined in the next section. To do this we first prove the following lemma which is a generalization of a well-known result in [8, p. 57].

LEMMA 5. If $u_{n} \rightarrow 0$ in $X$, then

(i) $\lim _{n \rightarrow \infty} i^{*} B u_{n}(t)=0$ in $V^{\prime}$ for each $t \in[0, T)$,

(ii) $\lim _{n \rightarrow \infty}\left\langle B u_{n}, u_{n}\right\rangle=0$ in $L^{1}(0, T)$,

(iii) $\lim _{n \rightarrow \infty} u_{n}=0$ in $L^{2}(0, T ; H)$,

where each limit in (i)-(iii) refers to the strong topology of the space indicated.

Proof. We first note that $i^{*} B u(\cdot)$ is an absolutely continuous function with values in $V^{\prime}$ since $i^{*} B u_{n}$ and $\left(i^{*} B u_{n}\right)^{\prime}$ are both in $L^{2}\left(0, T ; V^{\prime}\right)$. Thus $i^{*} B u_{n}(t)$ is well defined and

$$
\begin{aligned}
i^{*} B u(t) & =-\frac{1}{s} \int_{t}^{t+s}\left(B u_{n}\right)^{\prime}(r)(t+s-r) d r+\frac{i^{*}}{s} \int_{t}^{t+s} B u_{n}(r) d r \\
& =U_{n}+i^{*} V_{n} .
\end{aligned}
$$

Thus, for a given $\varepsilon>0$, it follows that $\left\|U_{n}\right\|_{V^{\prime}} \leqq \varepsilon$ for all $n$ if $s$ is small enough. With this choice for $s$ and $w \in W$, we have

$$
\left|\left\langle V_{n}, w\right\rangle_{W^{\prime}, W}\right|=\left|\int_{0}^{T}\left\langle B(r) \frac{1}{s} \chi_{[t, t+s]}(r) w, u_{n}(r)\right\rangle_{W^{\prime} W} d r\right| .
$$

Since $B(\cdot)(1 / s) \chi_{[t, t+s]}(\cdot) \in L^{2}\left(0, T ; W^{\prime}\right)$ and $u_{n} \rightarrow 0$ in $L^{2}(0, T ; V)$, the right-hand side of (3.5) converges to 0 . But $w \in W$ was arbitrary and therefore $V_{n} \rightarrow 0$ in $W^{\prime}$. The inclusion map of $V$ into $W$ is compact and thus $i^{*} V_{n}$ converges strongly to 0 in $V^{\prime}$. This proves (i) since $\varepsilon>0$ was arbitrary.

To prove (ii), let $\varepsilon>0$ be given. If $\alpha$ is large enough, we have

$$
\begin{aligned}
\int_{0}^{T}\left\langle B u_{n}(t), u_{n}(t)\right\rangle d t & \leqq \frac{\alpha^{2}}{2} \int_{0}^{T}\left\|i^{*} B u_{n}(t)\right\|_{V^{\prime}}^{2} d t+\frac{1}{2 \alpha^{2}} \int_{0}^{T}\left\|u_{n}(t)\right\|_{V}^{2} d t \\
& \leqq \varepsilon+\frac{\alpha^{2}}{2} \int_{0}^{T}\left\|i^{*} B u_{n}(t)\right\|_{V^{\prime}}^{2} d t .
\end{aligned}
$$

In view of Lemma 1 , the term $\left\|i^{*} B u_{n}(t)\right\|_{V^{\prime}}^{2}$ is bounded independently of $t$ and $n$. Therefore, the Dominated Convergence Theorem [9] and (i) imply the convergence to zero of the last term of (3.6), and since $\varepsilon$ was arbitrary, part (ii) follows. Part (iii) is clearly implied by (ii). This completes the proof of the lemma.

As a final step in establishing the validity of (2.4) and (2.6), we introduce the definitions

(i) $\quad A(v)=\tilde{A}(v+w)$,

(ii) $f(v)=-\tilde{A}(w+v) w-i^{*} B^{\prime} w-i^{*} B w^{\prime}+g$,

where $w$ and $w^{\prime}$ are both in $L^{2}\left(0, T ; H^{2}(\Omega)\right), v \in L^{2}(0, T ; V), g \in L^{2}\left(0, T ; V^{\prime}\right)$ and $i$ is the injection map of $V$ into $H^{2}(\Omega)$. Then the following lemma can be established.

LemMA 6. Hypotheses (2.4) and (2.6) hold.

Proof. By $(3.1)_{4}$, it is clear that there exists $Q<\infty$ such that $\|A(u)\| \leqq Q$ for all $u \in L^{2}(0, T ; V)$. Now let $u_{n} \rightarrow u$ in $X$ and let $v_{n} \rightarrow v$ in $L^{2}(0, T ; V)$. From Lemma 5 , 
$\lim _{n \rightarrow \infty}\left\|u_{n}-u\right\|_{L^{2}(0, T ; H)}=0$. Therefore a subsequence of $\left\{u_{n}\right\}$ converges to $u$ a.e. in $t$ and $x$. Then (2.4) follows from the Dominated Convergence Theorem and (3.1) $)_{5}$. Hypothesis (2.6) also holds by similar arguments.

In view of the above arguments, we have reduced the problem of existence of solutions to the abstract evolution equation (2.7) in the special context of $\S 3$ to the verification of the coercivity inequality (2.5). This will be discussed in the next section. For the convenience of presentation, however, this inequality will be assumed to hold in the remaining part of this section in order to provide the explicit form of the partial differential equation that we are concerned with here.

To do this, we define $g \in L^{2}\left(0, T ; V^{\prime}\right)$ to be given by the relation

$$
\begin{gathered}
\langle g, v\rangle=\int_{0}^{T} \int_{\Omega} h(t)(x) v(t)(x) d x+\int_{\partial \Omega} l(t)(x) v(t)(x) d A \\
\left.\quad+\int_{\partial \Omega} k(t)(x) \frac{\partial v(t)}{\partial n}(x) d A\right] d t
\end{gathered}
$$

where $h \in L^{2}(0, T ; H),(k, l) \in L^{2}\left(0, T ; L^{2}(\partial \Omega)\right)$ and $\partial \Omega$ is assumed to be a smooth two-dimensional manifold. Since the trace map from $H^{1}(\Omega)$ to $L^{2}(\partial \Omega)$ is continuous, it is clear that $g$ is in $L^{2}\left(0, T ; V^{\prime}\right)$. On assuming that $(2.5)$ holds, it follows that Theorem 1 implies the existence of $u \in X$ satisfying the equation

$$
\begin{aligned}
& -\int_{0}^{T} \int_{\Omega}\left[u(t)(x) v(x)+\tilde{D}_{i j}(x, t) \partial_{i} u(t)(x) \partial_{j} v(x)\right] d x \phi^{\prime}(t) d t \\
& +\int_{0}^{T} \int_{\Omega} D_{i j}(x, t ; w(t)(x)+u(t)(x)) \partial_{i} u(t)(x) \partial_{j} v(t)(x) d x \phi(t) d t \\
& +\int_{0}^{T} \int_{\Omega} E_{\alpha \beta}(x, t ; w(t)(x)+u(t)(x)) D^{\alpha} u(t)(x) D^{\beta} v(x) d x \phi(t) d t \\
& =\int_{0}^{T} \int_{\Omega} h(t)(x) v(x) d x \phi(t) d t \\
& +\int_{0}^{T} \int_{\partial \Omega}\left(l(t)(x) v(x)+k(t)(x) \frac{\partial v(x)}{\partial n}\right) d A \phi(t) d t \\
& -\int_{0}^{T} \int_{\Omega} D_{i j}(x, t ; w(t)(x)+u(t)(x)) \partial_{i} w(t)(x) \partial_{j} v(x) d x \phi(t) d t \\
& -\int_{0}^{T} \int_{\Omega} E_{\alpha \beta}(x, t ; w(t)(x)+u(t)(x)) D^{\alpha} w(t)(x) D^{\alpha} v(x) d x \phi(t) d t \\
& +\int_{0}^{T} \int_{\Omega}\left[w(t)(x) v(x)+\tilde{D}_{i j}(x, t) \partial_{i} w(t)(x) \partial_{j} v(x)\right] d x \phi^{\prime}(t) d t
\end{aligned}
$$

together with the initial condition

$$
i^{*} B u(0)=i^{*} B(0)\left(u_{0}-w(0)\right)
$$

for all $v \in V$ and $\phi \in C_{0}^{\infty}(0, T)$ provided $u_{0}-w(0) \in V$.

On restricting $v$ to be in $C_{0}^{\infty}(\Omega)$ and letting $z=u+w$ we see that a measurable representative of $z$ is a weak solution of the partial differential equation (0.6) subject to the initial condition $i^{*} B z(0)=i^{*} B(0) u_{0}$. Stable boundary conditions are obtained by properly selecting the space $V$, while variational boundary conditions are obtained 
by the use of the divergence theorem in (3.9). This leads to the formulation of a variety of initial-boundary value problems, representative examples of which are considered in the next section.

4. Boundary value problems. In this section we consider particular initial-boundary value problems pertaining to (0.6) and establish existence of weak solutions by utilizing the results derived earlier. As mentioned previously, our task has been reduced to the verification of the coercivity inequality (2.5). Here this is accomplished in relation to specific forms of the associated boundary conditions. Three different sets of such conditions are considered below. The first set corresponds to Dirichlet type and coercivity is obtained as a result of Garding's inequality. The other two examples include variational-type boundary conditions and coercivity is established by other means.

4.1. Dirichlet boundary conditions. We choose $V=H_{0}^{2}(\Omega)$ and assume that the coefficients $E_{\alpha \beta}$ for $|\alpha|=|\beta|=2$ are independent of $\rho$ and are continuous on $\bar{\Omega} \times[0, T]$. Moreover, we suppose that they obey the strong ellipticity condition

$$
\sum_{|\alpha|,|\beta|=2} E_{\alpha \beta}(x, t) \xi^{\alpha} \xi^{\beta} \geqq C|\xi|^{4} \text { for all } \xi \in \mathbb{R}^{3},
$$

so that the conditions of Garding's inequality [5] are satisfied. It then follows that (2.5) holds.

Thus, we have obtained existence of a weak solution to (1.6), denoted by $z$, along with boundary and initial conditions of the form

$$
\begin{aligned}
& z(t)-w(t) \in H_{0}^{2}(\Omega) \text { a.e., } \\
& i^{*} B z(0)=i^{*} B(0) u_{0},
\end{aligned}
$$

where $i$ is the injection map of $V$ into $W$. In less abstract fashion, the boundary condition $(4.2)_{1}$ can be expressed as

$$
\begin{aligned}
& z(t, x)=w(t, x), \quad x \in \partial \Omega, \\
& \partial_{i} z(t, x)=\partial_{i} w(t, x), \quad x \in \partial \Omega,
\end{aligned}
$$

where $w$ is the prescribed function defined earlier. Roughly speaking, (4.3) suggests that in contrast to second-order problems, both the function and its derivatives need to be specified on the boundary for this class of fourth-order problems. These problems may be viewed as pertinent to the later stages of the important metallurgical process of spinodal decomposition, where nonlinear effects dominate.

4.2. Variational boundary conditions. In discussing boundary conditions of variational type, we consider a simplified form of the diffusion equation (0.6) as follows:

$$
\frac{\partial}{\partial t}(\rho-\Delta \rho)-\partial_{i}\left(D(\rho) \partial_{i} \rho\right)+\Delta^{2} \rho=h
$$

This corresponds to assuming that the stress coefficient $c_{3}(\rho)$ in (1.4) is a constant and the mobility coefficient $\underset{\sim}{\alpha}$ in $(1.1)_{2}$ is a scalar $\alpha$. Physically, these assumptions mean that nonlinear effects are retained in the dependence of the usual diffusion coefficient $D$ but not in the small correcting terms, due to viscosity and surface tension.

We let $u_{0} \in V \equiv\left\{u \in H^{2}(\Omega)\right.$ such that $\partial u / \partial n=0$ on $\left.\partial \Omega\right\}$, with $\partial \Omega$ smooth. Then by the well-known theorem on elliptic regularity [10], $I-\Delta$ is a one-to-one and onto mapping from $V$ to $L^{2}(\Omega)$. It follows $(I-\Delta)^{-1}$ is continuous by the open mapping theorem [9]. Therefore, there exists a $K>0$ such that the following inequality holds:

$$
\|u\|_{V}=\left\|(I-\Delta)^{-1}(I-\Delta) u\right\|_{V} \leqq K\|(I-\Delta) u\|_{L^{2}(\Omega)} \leqq K\left(\|u\|_{L^{2}(\Omega)}+\|\Delta u\|_{L^{2}(\Omega)}\right) .
$$


Then $\langle A(v) u, y\rangle=\langle\tilde{A}(v+w) u, y\rangle$ is of the form

$$
\begin{gathered}
\int_{0}^{T} \int_{\Omega} D(v(t)(x)+w(t)(x)) \partial_{i} u(t)(x) \partial_{j} y(t)(x) d x d t \\
+\int_{0}^{T} \int_{\Omega} \Delta u(t)(x) \Delta y(t)(x) d x d t
\end{gathered}
$$

where $w(\cdot)$ and $w^{\prime}(\cdot)$ are both in $L^{2}\left(0, T ; H^{2}(\Omega)\right)$ and $D(\cdot)$ is bounded and continuous. Similarly, $\langle B(t) u, v\rangle$ is of the form

$$
\langle B(t) u, v\rangle=\int_{\Omega}(u v+\nabla u \cdot \nabla v) d x
$$

As a result of (4.5), it is easy to see through (4.6) and (4.7) that (2.5) holds. This establishes existence of solutions to (3.9) specialized to the present context. Applying then the divergence theorem, we obtain the existence of $u \in X$ such that $z=u+w$ satisfies (4.4) and the integral condition

$$
\int_{\partial \Omega} \frac{\partial}{\partial n}\left(z_{t}\right) v+D(z) \frac{\partial z}{\partial n} v+\Delta z \frac{\partial v}{\partial n}-v \frac{\partial(\Delta z)}{\partial n} d A=\int_{\partial \Omega}\left(l v+k \frac{\partial v}{\partial n}\right) d A
$$

for almost all values of $t$ and for all $v \in V$.

Therefore $z$ solves

$$
\frac{\partial}{\partial t}(z-\Delta z)-\partial_{i}\left(D(z) \partial_{i} z\right)+\Delta^{2} z=h,
$$

along with the initial condition

$$
\lim _{t \rightarrow 0+} \int_{\Omega}\left(z(t)-u_{0}\right) v+\nabla\left(z(t)-u_{0}\right) \cdot \nabla v d x=0 \quad \text { for all } v \in V
$$

and the boundary conditions

$$
\begin{aligned}
& \frac{\partial z(t, x)}{\partial n}=\frac{\partial w(t, x)}{\partial n} \quad \text { a.e. } t \text { and } x \in \partial \Omega, \\
& \frac{\partial z_{t}(t, x)}{\partial n}+D(z(t, x)) \frac{\partial z(t, x)}{\partial n}-\frac{\partial(\Delta z(t, x))}{\partial n}=l(t, x) \text { a.e. } t \text { and } x \in \partial \Omega
\end{aligned}
$$

where $(4.11)_{1}$ is stable resulting from the choice of $V$ and $(4.11)_{2}$ is of a variational type resulting from the divergence theorem.

The initial condition (4.10) can be expressed in a more conventional form by noting that for $u \in X, B u(t)$ is a function in $C\left(0, T ; W^{\prime}\right)$. It follows that $u \in C(0, T ; W)$ and thus $z=(u+w) \in C(0, T ; W)$. Therefore, the limit in (4.10) can be taken inside the integral giving

$$
\int_{\Omega}\left(z(0)-u_{0}\right) v+\nabla\left(z(0)-u_{0}\right) \cdot \nabla v d x=0 \quad \text { for all } v \in V .
$$

If $z(0)-u_{0} \in V$, it follows that the initial condition (4.12) takes the usual form,

$$
z(0, \cdot)=u_{0}(\cdot)
$$

The condition that $z(0, \cdot)-u_{0}(\cdot) \in V$ is equivalent to saying that $(\partial z / \partial n)(0, \cdot)=$ $(\partial w / \partial n)(0, \cdot)=\partial u_{0}(\cdot) / \partial n$ on $\partial \Omega$; that is, the initial condition $u_{0}(\cdot)$ and the boundary condition at $t=0, w(0, \cdot)$ are compatible. 
Next, we turn to a second example pertaining again to (4.4) but we now let $V=\left\{u \in H^{2}(\Omega)\right.$ such that $u(x)=0$ on $\left.\partial \Omega\right\}$. By reasoning similar to that of the previous example, (2.5) is again satisfied. Thus, in this case, we obtain the existence of a weak solution to the problem

$$
\begin{aligned}
& \frac{\partial}{\partial t}(z-\Delta z)-\partial_{i}\left(D(z) \partial_{i} z\right)+\Delta^{2} z=h, \\
& z(t, \cdot)=w(t, \cdot) \quad \text { on } \partial \Omega \text { for a.e. } t, \\
& \Delta z(t, \cdot)=k(t, \cdot) \quad \text { on } \partial \Omega \text { for a.e. } t, \\
& \lim _{t \rightarrow 0+} \int_{\Omega}\left(z(t)-u_{0}\right) v+\nabla\left(z(t)-u_{0}\right) \cdot \nabla v d x=0, \quad v \in V .
\end{aligned}
$$

As before, $z(\cdot)$ is in $C(0, T ; W)$ and if $z(0)-u_{0} \in V$, the initial condition $(4.14)_{4}$ takes the usual form $z(0, \cdot)=u_{0}(\cdot)$. In this case, the condition that $z(0)-u_{0} \in V$ is equivalent to the requirement that $u_{0}(\cdot)=w(0, \cdot)$ on $\partial \Omega$.

Other examples could be considered in a similar manner. Questions of existence of solutions to (0.6) or its specializations may thus be resolved by considering the verification of (2.5); that is the coercivity of a family of bilinear forms. This question of coercivity has been extensively studied and we refer to [10] for further discussion.

\section{REFERENCES}

[1] E. C. Aifantis, On the problem of diffusion in solids, Acta Mech., 37 (1980), pp. 265-296.

[2] T. W. Ting, Parabolic and pseudoparabolic partial differential equations, J. Math. Soc. Japan, 21 (1969), pp. 440-453.

[3] J. W. CAHN, On spinodal decomposition, Acta Metallurgica, 9 (1979), pp. 795-901.

[4] K. L. Kuttler AND E. C. Aifantis, Existence and uniqueness in nonclassical diffusion, Quart. Appl. Math., to appear.

[5] A. Friedman, Partial Differential Equations, Holt, Rinehart and Winston, New York, 1969.

[6] K. L. KutTler, Time dependent implicit evolution equations, Nonlinear Anal., 10 (1986), pp. 447-463.

[7] D. R. SMART, Fixed Point Theorems, Cambridge Univ. Press, 1974. (A generalization of the required fixed point theorem is stated on p. 32.)

[8] J. L. Lions, Quelques méthodes de résolution des problèmes aux limites non-lineares, Dunod, Paris, 1969.

[9] W. Rudin, Real and Complex Analysis, McGraw-Hill, New York, 1974.

[10] S. Agmon, Lectures on Elliptic Boundary Value Problems, Van Nostrand, New York, 1975.

[11] R. E. ShowAlTER, Degenerate evolution equations and applications, Indiana Univ. Math. J., 23 (1974), pp. 655-677. 